\title{
DAMPING FORCES EXERTED BY RAREFIED GAS MIXTURES IN MICRO-ELECTRO-MECHANICAL SYSTEM DEVICES VIBRATING AT HIGH FREQUENCIES
}

\author{
Marzia Bisi ${ }^{1} \mathcal{E}$ Silvia Lorenzani ${ }^{2, *}$ \\ ${ }^{1}$ Dipartimento di Matematica e Informatica, Università di Parma, Parco Area delle Scienze 53/A, \\ I-43124 Parma, Italy \\ ${ }^{2}$ Dipartimento di Matematica, Politecnico di Milano, Piazza Leonardo da Vinci 32, I-20133 \\ Milano, Italy \\ *Address all correspondence to Silvia Lorenzani, E-mail: silvia.lorenzani@polimi.it
}

\section{INTRODUCTION}

In the last few years, micro-electro-mechanical systems devices vibrating at high frequencies (RF MEMS) (ranging from $1 \mathrm{MHz}$ to $60 \mathrm{GHz}$ ) have increasingly been used in various industrial fields. Low-frequency MEMS devices are normally operated at very low pressure in order to minimize the damping due to the internal friction of the gas (viscous damping). This need can be overcome when MEMS devices vibrate at relatively high frequencies, since gas compressibility and inertial forces lead then to another damping mechanism which is related to the propagation of sound waves generated by high-frequency oscillating micro-structures. Very recently, it has been proved in Desvillettes and Lorenzani (2012) for a single-component gas that sound waves propagating between the micro-device walls induce a resonant/antiresonant response of the system. The occurrence of an antiresonance is particularly important since if the device is operated close to the corresponding frequency, the damping due to the gas is considerably reduced. Since gas resonances take place for each value of the rarefaction parameter (inverse Knudsen number), the RF MEMS devices can perform well also at atmospheric pressure, greatly reducing the need for (and cost associated with) vacuum packaging. In the current investigation, we extend the previous analysis carried out for a single species gas (Desvillettes and Lorenzani, 2012) to a gas mixture, by using the unsteady kinetic equation based on the linearized version of the relaxation model of BGK-type presented in Groppi and Spiga (2004). This BGK approximation has already been used as a consistent tool to investigate other physical problems, such as condensation and sublimation in gas mixtures (see Kosuge, Mizuno and Aoki, 2007, Kosuge, Aoki, Inoue, Goldstein and Varghese, 2012). The evaluation of damping forces exerted by different gases assumes a basic relevance in all MEMS fabrication processes. In fact, during the wafer bonding process a gas mixture of noble gases (like $\mathrm{Ar}, \mathrm{Kr}$, or $\mathrm{Ne}$ ) and getterable gases (like $\mathrm{N}_{2}, \mathrm{O}_{2}$, or $\mathrm{CO}_{2}$ ) is usually backfilled into the MEMS sensor package to set its operating pressure, which should exactly match the damping requirements of the micro-device design ("backfilling process"). 


\section{LINEARIZED BGK MODEL FOR A BINARY MIXTURE}

The evolution of a mixture of $N$ elastically scattering gases is usually described by a set of $N$ integro-differential equations of Boltzmann type for the species distribution functions $f^{s}(t, \mathbf{x}, \xi)(s=1, \ldots, N)$ (Chapman and Cowling, 1970). It is well known that suitably weighted integrals of $f^{s}$ over the molecular velocity $\xi$ provide the main macroscopic fields of physical interest, as number density $n^{s}$, mass velocity $\mathbf{v}^{s}$, temperature $T^{s}$, viscous stress tensor $P_{i j}^{s}$, heat flux $\mathbf{q}^{s}$. At global equilibrium, all distribution functions are Maxwellians

$$
f_{0}^{s}=n_{0}^{s}\left(\frac{m^{s}}{2 \pi k T_{0}}\right)^{3 / 2} \exp \left[-\frac{m^{s}}{2 k T_{0}}\left|\xi-\mathbf{v}_{\mathbf{0}}\right|^{2}\right]
$$

for some common velocity $\mathbf{v}_{0}$ and temperature $T_{0}$. Since Boltzmann equations are quite awkward to deal with, consistent simpler models have been proposed in the literature. Among them, the McCormack model (1973) has been over the years the most widely used to study a great variety of problems, such as Couette and Poiseuille flows, thermal creep, and heat transfer for mixtures, since all transport coefficients (i.e., viscosity, thermal conductivity, diffusion, and thermal diffusion ratio) can be correctly obtained from it applying the Chapman-Enskog procedure. Moreover, McCormack derived a model for the cross-collision operator of a general multicomponent monatomic mixture, not restricted to Maxwell molecules, but applicable also to other intermolecular force laws. Instead, in the present paper we have chosen to perform our numerical analysis by using the BGK relaxation model proposed in Andries et al. (2002) and generalized in Groppi and Spiga (2004) even to nonconservative collisions, that has been proven to be well posed from the mathematical point of view (correct Boltzmann collision invariants and Maxwellian equilibria are properly recovered, and $H$ theorem is fulfilled). Even if the BGK-Boltzmann equation for mixtures generally cannot match all the transport coefficients simultaneously (as it occurs for a single-component gas BGK-model), for the specific problem at hand, the BGK equation has been shown to be a valuable tool for investigation, at least in the case of a pure monatomic gas (Desvillettes and Lorenzani, 2012), giving results in close agreement with those obtained with more refined kinetic models. Furthermore, the use of the BGK model equation allows one to detect, by a simple visual inspection, if the equations derived for describing the sound wave propagation in a gaseous mixture are formally correct, since when the binary mixture reduces to a single gas, one should recover the same equations reported in Desvillettes and Lorenzani (2012).

For a mixture of two gases with particle masses $m^{1}, m^{2}$, the BGK model presented in Groppi and Spiga (2004) reads as

$$
\frac{\partial f^{s}}{\partial t}+\xi \cdot \nabla_{\mathbf{x}} f^{s}=v_{s}\left(\mathcal{M}_{s}-f^{s}\right) \quad s=1,2
$$

where $v_{s}$ are suitable collision frequencies (independent from $\xi$ ) and $\mathcal{M}_{s}$ are Maxwellian attractors

$$
\mathcal{M}_{s}=n^{s}\left(\frac{m^{s}}{2 \pi k T_{s}}\right)^{3 / 2} \exp \left[-\frac{m^{s}}{2 k T_{s}}\left|\xi-\mathbf{v}_{s}\right|^{2}\right]
$$

Auxiliary parameters $\mathbf{v}_{s}, T_{s}$ (with subscript $s$ ) are determined in terms of the moments of the distribution functions $f^{s}$, that is $n^{s}, \mathbf{v}^{s}, T^{s}$ (with superscript $s$ ), by imposing that the exchange rates for species momenta and energies given by the BGK operator reproduce the exact corresponding rates calculated by the Boltzmann operators for Maxwell molecules. Skipping intermediate details, their final expressions written in compact form (for $s=1,2$ ) are

$$
\begin{gathered}
\mathbf{v}_{s}=\mathbf{v}^{s}+(-1)^{s} \frac{v_{1}^{12}}{v_{s}} \frac{\mu^{12}}{m^{s}} \frac{n^{1} n^{2}}{n^{s}}\left(\mathbf{v}^{1}-\mathbf{v}^{2}\right) \\
k T_{s}=k T^{s}-(-1)^{s} \frac{2}{3} \frac{v_{1}^{12}}{v_{s}} \mu^{12} \frac{n^{1} n^{2}}{n^{s}} \mathbf{v}^{s} \cdot\left(\mathbf{v}^{1}-\mathbf{v}^{2}\right)-\frac{1}{3 m^{s}}\left(\frac{v_{1}^{12}}{v_{s}} \mu^{12} \frac{n^{1} n^{2}}{n^{s}}\right)^{2}\left|\mathbf{v}^{1}-\mathbf{v}^{2}\right|^{2} \\
+2(-1)^{s} \frac{v_{1}^{12}}{v_{s}} \frac{\mu^{12}}{\left(m^{1}+m^{2}\right)} \frac{n^{1} n^{2}}{n^{s}} k\left(T^{1}-T^{2}\right)+\frac{2}{3}(-1)^{s} \frac{v_{1}^{12}}{v_{s}} \frac{\mu^{12}}{\left(m^{1}+m^{2}\right)} \frac{n^{1} n^{2}}{n^{s}}\left(m^{1} \mathbf{v}^{1}+m^{2} \mathbf{v}^{2}\right)\left(\mathbf{v}^{1}-\mathbf{v}^{2}\right)
\end{gathered}
$$


where $\mu^{12}=m^{1} m^{2} /\left(m^{1}+m^{2}\right)$ stands for the reduced mass, and $v_{k}^{s r}$ are suitably weighted integrals of the differential cross section $\sigma^{s r}$ relevant to collisions between particles of species $s$ and $r$ :

$$
v_{k}^{s r}=2 \pi \int_{0}^{\pi} \sigma^{s r}(\chi)\left(1-\cos ^{k} \chi\right) \sin \chi d \chi
$$

whose values for intermolecular potentials of inverse power type are reported in Chapman and Cowling (1970). For the special case of the Maxwell molecule interparticle force law

$$
F^{s r}=\frac{K^{s r}}{r_{d}^{5}}
$$

where $r_{d}$ is the distance between particles and $K^{s r}$ is the interparticle force law constant, the microscopic collision frequencies, relevant for the present analysis, are explicitly given by

$$
\begin{aligned}
& v_{1}^{12}=2 \pi A_{1}(5)\left(\frac{K^{12}}{\mu^{12}}\right)^{1 / 2} \\
& v_{2}^{12}=2 \pi A_{2}(5)\left(\frac{K^{12}}{\mu^{12}}\right)^{1 / 2} \\
& v_{2}^{11}=2 \pi A_{2}(5)\left(\frac{2 K^{11}}{m^{1}}\right)^{1 / 2} \\
& v_{2}^{22}=2 \pi A_{2}(5)\left(\frac{2 K^{22}}{m^{2}}\right)^{1 / 2}
\end{aligned}
$$

with $A_{1}(5) \simeq 0.422, A_{2}(5) \simeq 0.436$ being the dimensionless collision cross sections reported in Chapman and Cowling (1970). In a binary mixture, $K^{11}$ and $K^{22}$ can be written in terms of the simple gas viscosity coefficients $\eta^{s}$ $(s=1,2)$ with aid of the first Chapman-Enskog expressions for these quantities:

$$
\eta^{s}=\frac{1}{3 \pi}\left(\frac{2 m^{s}}{K^{s s}}\right)^{1 / 2} \frac{(k T)}{A_{2}(5)} .
$$

In order to specify the force constants $K^{11}$ and $K^{22}$, the experimental data on the viscosities $\eta^{s}$ of the single gases at the temperature $T=300 \mathrm{~K}$, given in Kestin et al. (1984), have been used. Finally, the constant $K^{12}$ has been determined from the method of the combination rule

$$
K^{12}=\left(K^{11} \cdot K^{22}\right)^{1 / 2}
$$

previously considered by McCormack (1973).

A useful tool in the sequel will be the linearization of BGK equations (2) around the equilibrium configuration given by Eq. (1), where we have assumed that $\mathbf{v}_{0}=0$ without loss of generality. In particular, we set $f^{s}=f_{0}^{s}\left(1+h^{s}\right)$ with $\left|h^{s}\right| \ll 1$, and we neglect higher order terms. It can be easily checked that

$$
\begin{gathered}
n^{s}=n_{0}^{s}+\int_{\mathbf{R}^{3}} f_{0}^{s} h^{s} d \xi \\
\mathbf{v}^{s}=\frac{1}{n_{0}^{s}} \int_{\mathbf{R}^{3}} \xi f_{0}^{s} h^{s} d \xi \\
T^{s}=T_{0}+\frac{m^{s}}{3 k n_{0}^{s}} \int_{\mathbf{R}^{3}}\left(|\xi|^{2}-\frac{3 k}{m^{s}} T_{0}\right) f_{0}^{s} h^{s} d \xi .
\end{gathered}
$$


Introducing now the normalizations

$$
\begin{gathered}
\mathbf{c}=\frac{\xi}{\sqrt{2 \frac{k}{m^{1}} T_{0}}} \\
\hat{f}_{0}^{1}=\frac{f_{0}^{1}}{n_{0}^{1}}=\frac{\mathrm{e}^{-|\mathbf{c}|^{2}}}{\pi^{3 / 2}} \\
\hat{f}_{0}^{2}=\frac{f_{0}^{2}}{n_{0}^{2}}=\frac{\mathrm{e}^{-\frac{|\mathbf{c}|^{2}}{M_{12}}}}{\left(\pi M_{12}\right)^{3 / 2}}
\end{gathered}
$$

(where $M_{12}=m^{1} / m^{2}$ is the mass ratio) we get the linearized version of the BGK collision term, given by the right-hand side of Eq. (2):

$$
\begin{aligned}
L h^{1}{ }_{B G K} & =v_{1}\left\{\rho^{1}+2\left(1-\Gamma^{1}\right) \mathbf{c} \cdot \mathbf{v}^{1}+2 \Gamma^{1} \mathbf{c} \cdot \mathbf{v}^{2}+\left[1-\frac{2 \Gamma^{1} M_{12}}{\left(1+M_{12}\right)}\right]\left(c^{2}-\frac{3}{2}\right) \tau^{1}\right. \\
& \left.+\frac{2 \Gamma^{1} M_{12}}{\left(1+M_{12}\right)}\left(c^{2}-\frac{3}{2}\right) \tau^{2}-h^{1}\right\} \\
L h_{B G K}^{2} & =v_{2}\left\{\rho^{2}+2\left(1-\Gamma^{2}\right) \frac{\mathbf{c}}{M_{12}} \cdot \mathbf{v}^{2}+2 \Gamma^{2} \frac{\mathbf{c}}{M_{12}} \cdot \mathbf{v}^{1}+\left[1-\frac{2 \Gamma^{2}}{\left(1+M_{12}\right)}\right]\left(\frac{c^{2}}{M_{12}}-\frac{3}{2}\right) \tau^{2}\right. \\
+ & \left.\frac{2 \Gamma^{2}}{\left(1+M_{12}\right)}\left(\frac{c^{2}}{M_{12}}-\frac{3}{2}\right) \tau^{1}-h^{2}\right\}
\end{aligned}
$$

where $\Gamma^{1}=\left(v_{1}^{12} \mu^{12}\right) /\left(v_{1} m^{1}\right) n_{0}^{2}, \Gamma^{2}=\left(v_{1}^{12} \mu^{12}\right) /\left(v_{2} m^{2}\right) n_{0}^{1}$, while $\rho^{1}, \rho^{2}, \mathbf{v}^{1}, \mathbf{v}^{2}, \tau^{1}, \tau^{2}$ are the macroscopic fields for the two gas components given by Eqs. (25)-(30). The values of collision frequencies $v_{1}, v_{2}$ are determined by imposing, as additional constraint, that the (linearized) BGK model reproduces the (linearized) exchange rates for viscous stress tensors $P_{i j}^{1}$ and $P_{i j}^{2}$ prescribed by Boltzmann equations. Skipping calculation details, such a constraint yields

$$
\begin{aligned}
& v_{1}=\frac{3}{4} v_{2}^{11} n_{0}^{1}+\frac{3}{2} v_{2}^{12} \frac{1}{1+M_{12}} n_{0}^{2} \\
& v_{2}=\frac{3}{2} v_{2}^{12} \frac{M_{12}}{1+M_{12}} n_{0}^{1}+\frac{3}{4} v_{2}^{22} n_{0}^{2} .
\end{aligned}
$$

\section{PROBLEM FORMULATION}

Let us consider a binary gaseous mixture confined between two flat, infinite, and parallel plates located at $z^{\prime}=-d / 2$ and $z^{\prime}=d / 2$. Both boundaries are held at the same constant temperature. The upper wall of the channel (located at $z^{\prime}=d / 2$ ) is fixed while the lower one (located at $z^{\prime}=-d / 2$ ) harmonically oscillates in the $z^{\prime}$ direction (normal to the wall itself) with angular frequency $\omega^{\prime}$ (the corresponding period being $T^{\prime}=2 \pi / \omega^{\prime}$ ). The velocity $U_{w}^{\prime}$ of the oscillating plate depends on time $t^{\prime}$ through the formula: $U_{w}^{\prime}\left(t^{\prime}\right)=U_{0}^{\prime} \sin \left(\omega^{\prime} t^{\prime}\right)$, where it is assumed that the amplitude $U_{0}^{\prime}$ is very small compared to the characteristic molecular velocity of the mixture given by $v_{0}=\sqrt{2 k T_{0} / m}$, with $k$ being the Boltzmann constant, $m$ being the mean molecular mass of the mixture, and $T_{0}$ being the equilibrium temperature of the mixture. Under these conditions, the Boltzmann equation modeling the gaseous mixture motion inside the channel can be linearized by representing the distribution function of each species, $f^{s}$, as mentioned in the previous section. Then, the system of the BGK-Boltzmann equations after the projection (Desvillettes and Lorenzani, 2012) reads

$$
\begin{aligned}
\frac{\partial H^{1}}{\partial t}+c_{z} \frac{\partial H^{1}}{\partial z}+H^{1} & =\rho^{1}+2\left(1-\Gamma^{1}\right) c_{z} v_{z}^{1}+2 \Gamma^{1} c_{z} v_{z}^{2}+\left[1-\frac{2 \Gamma^{1} M_{12}}{\left(1+M_{12}\right)}\right]\left(c_{z}^{2}-\frac{1}{2}\right) \tau^{1} \\
& +\frac{2 \Gamma^{1} M_{12}}{\left(1+M_{12}\right)}\left(c_{z}^{2}-\frac{1}{2}\right) \tau^{2}
\end{aligned}
$$




$$
\begin{aligned}
& \frac{\partial H^{2}}{\partial t}+c_{z} \frac{\partial H^{2}}{\partial z}+\Theta_{12} H^{2}=\Theta_{12}\left\{\rho^{2}+\frac{2\left(1-\Gamma^{2}\right)}{M_{12}} c_{z} v_{z}^{2}+\frac{2 \Gamma^{2}}{M_{12}} c_{z} v_{z}^{1}+\left[1-\frac{2 \Gamma^{2}}{\left(1+M_{12}\right)}\right]\left(\frac{c_{z}^{2}}{M_{12}}-\frac{1}{2}\right) \tau^{2}\right. \\
&\left.+\frac{2 \Gamma^{2}}{\left(1+M_{12}\right)}\left(\frac{c_{z}^{2}}{M_{12}}-\frac{1}{2}\right) \tau^{1}\right\} \\
& \frac{\partial \Psi^{1}}{\partial t}+c_{z} \frac{\partial \Psi^{1}}{\partial z}+\Psi^{1}= {\left[1-\frac{2 \Gamma^{1} M_{12}}{\left(1+M_{12}\right)}\right] \tau^{1}+\frac{2 \Gamma^{1} M_{12}}{\left(1+M_{12}\right)} \tau^{2} } \\
& \frac{\partial \Psi^{2}}{\partial t}+c_{z} \frac{\partial \Psi^{2}}{\partial z}+\Theta_{12} \Psi^{2}=\Theta_{12}\left\{\left[1-\frac{2 \Gamma^{2}}{\left(1+M_{12}\right)}\right] \tau^{2}+\frac{2 \Gamma^{2}}{\left(1+M_{12}\right)} \tau^{1}\right\}
\end{aligned}
$$

where all variables have been rescaled as follows: $t=t^{\prime} / \theta_{1}, z=z^{\prime} /\left(v_{0}^{1} \theta_{1}\right)$ with $v_{0}^{1}=\sqrt{2 k T_{0} / m^{1}}$, and $\Theta_{12}=$ $\theta_{1} / \theta_{2}=v_{2} / v_{1}, \delta=d /\left(v_{0}^{1} \theta_{1}\right)$ (which is the dimensionless distance between the channel walls as well as the rarefaction parameter of the species $s=1$ ). Notice that in the particular case $M_{12}=1$, namely if the two gases have equal particle mass, the equations for the pair $\left(H^{1}, \Psi^{1}\right)$ coincide with the ones for $\left(H^{2}, \Psi^{2}\right)$ only if $\Gamma^{1}=\Gamma^{2}$ and $\Theta_{12}=1$, hence only if $n_{0}^{1}=n_{0}^{2}$ and the two gases share even the same viscosity $\eta^{1}=\eta^{2}$. In Eqs. (17)-(20), the reduced unknown distribution functions $H^{s}$ and $\Psi^{s}$ are defined as

$$
\begin{gathered}
H^{1}\left(z, c_{z}, t\right)=\frac{1}{\pi} \int_{-\infty}^{+\infty} \int_{-\infty}^{+\infty} h^{1}(z, \mathbf{c}, t) e^{-\left(c_{x}^{2}+c_{y}^{2}\right)} d c_{x} d c_{y} \\
H^{2}\left(z, c_{z}, t\right)=\frac{1}{\pi M_{12}} \int_{-\infty}^{+\infty} \int_{-\infty}^{+\infty} h^{2}(z, \mathbf{c}, t) e^{-\left(c_{x}^{2}+c_{y}^{2}\right) / M_{12}} d c_{x} d c_{y} \\
\Psi^{1}\left(z, c_{z}, t\right)=\frac{1}{\pi} \int_{-\infty}^{+\infty} \int_{-\infty}^{+\infty}\left(c_{x}^{2}+c_{y}^{2}-1\right) h^{1}(z, \mathbf{c}, t) e^{-\left(c_{x}^{2}+c_{y}^{2}\right)} d c_{x} d c_{y} \\
\Psi^{2}\left(z, c_{z}, t\right)=\frac{1}{\pi M_{12}} \int_{-\infty}^{+\infty} \int_{-\infty}^{+\infty}\left(\frac{c_{x}^{2}+c_{y}^{2}}{M_{12}}-1\right) h^{2}(z, \mathbf{c}, t) e^{-\left(c_{x}^{2}+c_{y}^{2}\right) / M_{12}} d c_{x} d c_{y}
\end{gathered}
$$

and the macroscopic fields as

$$
\begin{gathered}
\rho^{1}(z, t)=\frac{1}{\sqrt{\pi}} \int_{-\infty}^{+\infty} H^{1} e^{-c_{z}^{2}} d c_{z} \\
\rho^{2}(z, t)=\frac{1}{\sqrt{\pi M_{12}}} \int_{-\infty}^{+\infty} H^{2} e^{-c_{z}^{2} / M_{12}} d c_{z} \\
v_{z}^{1}(z, t)=\frac{1}{\sqrt{\pi}} \int_{-\infty}^{+\infty} c_{z} H^{1} e^{-c_{z}^{2}} d c_{z} \\
v_{z}^{2}(z, t)=\frac{1}{\sqrt{\pi M_{12}}} \int_{-\infty}^{+\infty} c_{z} H^{2} e^{-c_{z}^{2} / M_{12}} d c_{z} \\
\tau^{1}(z, t)=\frac{1}{\sqrt{\pi}} \int_{-\infty}^{+\infty} \frac{2}{3}\left[\left(c_{z}^{2}-1 / 2\right) H^{1}+\Psi^{1}\right] e^{-c_{z}^{2}} d c_{z} \\
\tau^{2}(z, t)=\frac{1}{\sqrt{\pi M_{12}}} \int_{-\infty}^{+\infty} \frac{2}{3}\left[\left(\frac{c_{z}^{2}}{M_{12}}-\frac{1}{2}\right) H^{2}+\Psi^{2}\right] e^{-c_{z}^{2} / M_{12}} d c_{z}
\end{gathered}
$$

Assuming the diffuse scattering of gaseous particles on both walls, the linearized boundary conditions read as

$$
H^{1}\left(z=-\delta / 2, c_{z}, t\right)=\left(\sqrt{\pi}+2 c_{z}\right) U_{w}-2 \int_{\tilde{c}_{z}<0} d \tilde{c}_{z} \tilde{c}_{z} e^{-\tilde{c}_{z}^{2}} H^{1}\left(z=-\delta / 2, \tilde{c}_{z}, t\right), \quad c_{z}>0
$$




$$
\begin{gathered}
H^{2}\left(z=-\delta / 2, c_{z}, t\right)=\left(\sqrt{\frac{\pi}{M_{12}}}+\frac{2 c_{z}}{M_{12}}\right) U_{w}-\frac{2}{M_{12}} \int_{\tilde{c}_{z}<0} d \tilde{c}_{z} \tilde{c}_{z} e^{-\tilde{c}_{z}^{2} / M_{12}} H^{2}\left(z=-\delta / 2, \tilde{c}_{z}, t\right), \quad c_{z}>0 \\
\Psi^{1}\left(z=-\delta / 2, c_{z}, t\right)=\Psi^{2}\left(z=-\delta / 2, c_{z}, t\right)=0, \quad c_{z}>0 \\
H^{1}\left(z=\delta / 2, c_{z}, t\right)=2 \int_{\tilde{c}_{z}>0} d \tilde{c}_{z} \tilde{c}_{z} e^{-\tilde{c}_{z}^{2}} H^{1}\left(z=\delta / 2, \tilde{c}_{z}, t\right), \quad c_{z}<0 \\
H^{2}\left(z=\delta / 2, c_{z}, t\right)=\frac{2}{M_{12}} \int_{\tilde{c}_{z}>0} d \tilde{c}_{z} \tilde{c}_{z} e^{-\tilde{c}_{z}^{2} / M_{12}} H^{2}\left(z=\delta / 2, \tilde{c}_{z}, t\right), \quad c_{z}<0 \\
\Psi^{1}\left(z=\delta / 2, c_{z}, t\right)=\Psi^{2}\left(z=\delta / 2, c_{z}, t\right)=0, \quad c_{z}<0
\end{gathered}
$$

In Eqs. (31) and (32), $U_{w}$ is the dimensionless wall velocity given by $U_{w}(t)=U_{0} \sin (\omega t)$ with $U_{w}=U_{w}^{\prime} / v_{0}^{1}$, $U_{0}=U_{0}^{\prime} / v_{0}^{1}, \omega=\theta_{1} \omega^{\prime}, T=2 \pi / \omega=T^{\prime} / \theta_{1}$. The time-dependent problem described by Eqs. (17)-(20), with boundary conditions given by Eqs. (31)-(36), has been numerically solved by a deterministic finite-difference method. The region between the plates has been divided into a number of cells of the same size. In MEMS devices vibrating at high frequencies, high spatial resolution is required in order to capture properly the propagation of sound waves with short wavelength. Following previous numerical studies (see Lorenzani et al., 2007), the emitter plate displacement is not taken into account. The gas feels the plate motion only through the boundary conditions (31)-(36). Similarly, a regular net of nodes has been arranged into a finite domain of the one-dimensional velocity space, large enough to contain the significant part of the distribution functions at any spatial location. In general, the extent of the velocity distribution function of species $s(s=1,2)$ in the molecular velocity space is inversely proportional to $\sqrt{m^{s}}$. Thus, a separate grid for each species should be considered. Indeed, due to the normalization adopted in the present paper [Eq. (10)], we were able to use only one grid in the velocity space since the scaling factor: $\sqrt{m^{1} / m^{2}}=\sqrt{M_{12}}$ (which allows one to switch between the grid associated with the first species and that associated with the second species) appears directly in the equations defining the reduced distribution functions $H^{s}$ and $\Psi^{s}$ [Eqs. (21)-(24)] and the macroscopic fields [Eqs. (25)-(30)]. Then, the distribution functions are assumed to be constant within each cell of the phase space. Further details on the standard (upwind) implicit finite-difference scheme employed in the present computations are reported in Lorenzani et al. (2007). The convergence of the numerical results presented has been extensively examined by modifying the number of grid points chosen in phase space. The variation of the components of the macroscopic fields due to the increase of the grid parameters was the criterion of the calculation accuracy. In addition, it has been checked that, allowing our data to be reduced to the case of a single monoatomic gas by taking $m^{1}=m^{2}$ and $\eta^{1}=\eta^{2}$, the results presented in Desvillettes and Lorenzani (2012) can be recovered.

In order to compute the damping force exerted by the gaseous mixture on the moving wall of the channel, the global normal stress $P_{z z}$ has to be evaluated at $z=-\delta / 2$. In the frame of our linearized analysis, the normal component of the stress tensor of the mixture is defined in terms of the single component parameters as $P_{z z}=P_{z z}^{1}+P_{z z}^{2}$, where

$$
\begin{gathered}
P_{z z}^{1}(z, t)=\frac{1}{\sqrt{\pi}} \int_{-\infty}^{+\infty} c_{z}^{2} H^{1} e^{-c_{z}^{2}} d c_{z} \\
P_{z z}^{2}(z, t)=\frac{1}{\sqrt{\pi M_{12}}} \int_{-\infty}^{+\infty} c_{z}^{2} H^{2} e^{-c_{z}^{2} / M_{12}} d c_{z}
\end{gathered}
$$

The normal stress time dependence is of the known form

$$
\left|P_{z z}\right| \sin (\omega t+\phi)
$$

where $\left|P_{z z}\right|$ is the amplitude and $\phi$ the phase. In general, the amplitude of the time-dependent macroscopic fields is extracted from our numerical results as half the vertical distance between a maximum and the nearest minimum appearing in the temporal evolution of the macroscopic quantity. Since the parameter range investigated in our numerical simulations is close to the one taken into account in Desvillettes and Lorenzani (2012), about the accuracy of the BGK approximation we consider valid the same remarks reported in that reference for a single component gas (still lacking a complete experimental data set). 


\section{RESULTS AND DISCUSSION}

The numerical results presented here refer to the noble gaseous mixture of helium, $\mathrm{He}\left(m^{1}=4.0026\right.$ au), and xenon, $\mathrm{Xe}\left(m^{2}=131.29 \mathrm{au}\right)$ with the same molar concentration. For a single component gas, we have found in Desvillettes and Lorenzani (2012) that above a certain frequency of oscillation of the lower wall of the channel, the sound waves propagating through the gas are trapped in the gaps between the moving elements and the fixed boundaries of the microdevice. In particular, we found a scaling law (valid for all Knudsen numbers) that predicts a resonant response of the system when the dimensionless distance between the channel walls (measured in units of the oscillation period of the moving plate), $L=\delta / T$, takes a well-defined fixed value. Physically, the origin of this phenomenon can be traced back to the constructive interference (resonance) or destructive interference (antiresonance) occurring between the incident and reflected sound waves. Corresponding to a resonant response of the system, the amplitude of $P_{z z}$ at $z=-\delta / 2$ reaches its maximum value (resonance) or its minimum value (antiresonance). Beyond the phenomenon of sound wave resonances and the scaling law related to it, firstly reported in Desvillettes and Lorenzani (2012), the nonmonotonic dependence of the amplitude of $P_{z z}$ on the period $T$ has already been detected for a single-component gas in several previous papers (see Kalempa and Sharipov, 2009).

In Figs. 1-3, we report the profiles of the normal stress amplitude at the oscillating wall as a function of the period $T$, for three different values of the rarefaction parameter $\delta$, for a single component gas as well as for a He-Xe mixture. For future comparisons, we have also listed in Tables 1-3 the numerical values of the amplitude of $P_{z z}$ at $z=-\delta / 2$ as a function of the period $T$ for the $\mathrm{He}-\mathrm{Xe}$ mixture in different molecular flow regimes. The pictures reveal that in the near-free molecular flow regime $(\delta=0.1)$ and in the transitional region $(\delta=1)$ the resonant response of the system occurs also in the gas mixture (although the scaling law found for a single-component gas does not hold any longer), while in the near-continuum regime $(\delta=10)$ the propagation of sound waves in the He-Xe mixture does not show any resonance. In order to inspect more deeply the physical processes going on in a disparate-mass gas mixture, we report in Figs. 4 and 5 the velocities of gas components ( $v_{z}^{1}$ for helium and $v_{z}^{2}$ for xenon) in the $z$ direction against the distance across the gap of the channel (at different stages during a period of oscillation). The pictures reveal that for both the



FIG. 1: Amplitude of the normal stress tensor $P_{z z}$ at the oscillating wall versus $T$ for $\delta=0.1$. Comparison between the results obtained for a single-component gas (circles) and for the He-Xe mixture (squares). 




FIG. 2: Amplitude of the normal stress tensor $P_{z z}$ at the oscillating wall versus $T$ for $\delta=1$. Comparison between the results obtained for a single-component gas (circles) and for the He-Xe mixture (squares).

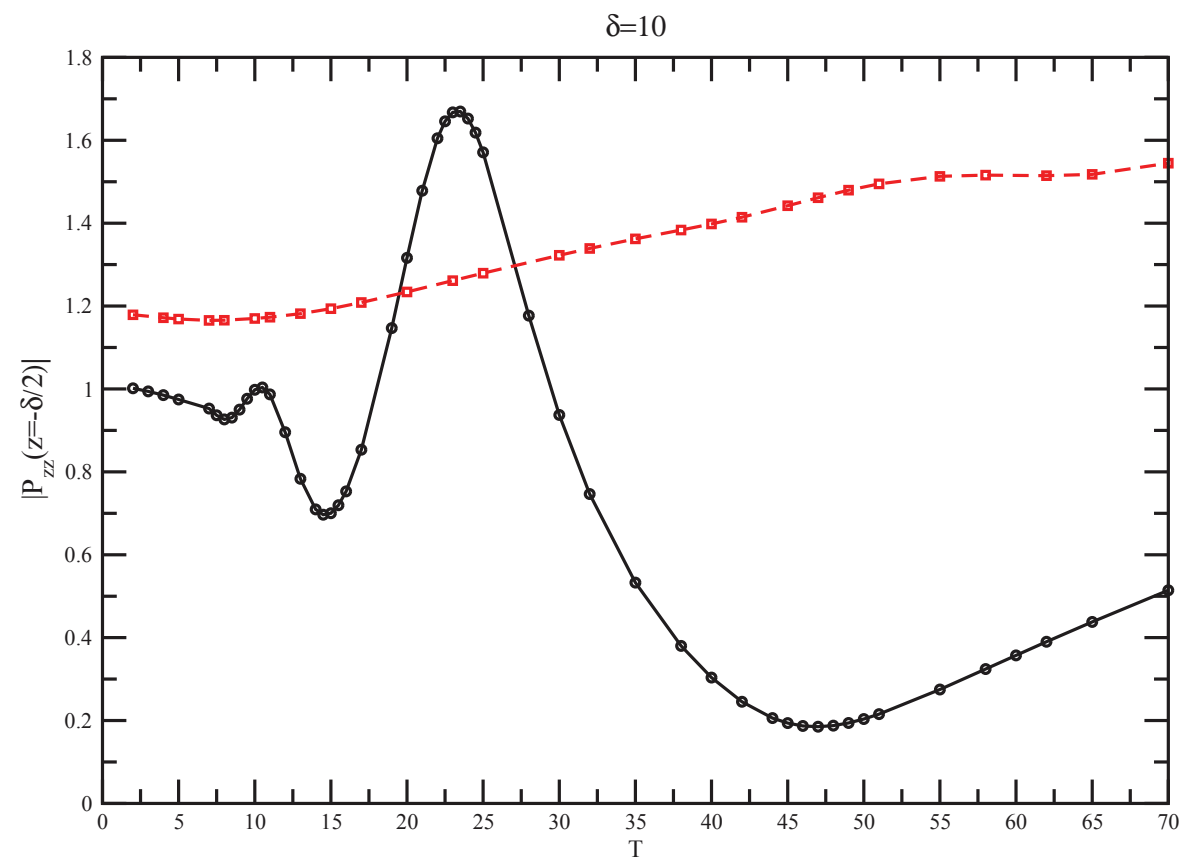

FIG. 3: Amplitude of the normal stress tensor $P_{z z}$ at the oscillating wall versus $T$ for $\delta=10$. Comparison between the results obtained for a single-component gas (circles) and for the He-Xe mixture (squares). 
TABLE 1: Amplitude of $P_{z z}$ at $z=-\delta / 2$ for the He-Xe mixture and $\delta=0.1$

\begin{tabular}{|c|c|}
\hline$T$ & $\left|P_{z z}(z=-\delta / 2)\right|$ \\
\hline 0.15 & 1.273 \\
\hline 0.20 & 1.432 \\
\hline 0.25 & 1.234 \\
\hline 0.30 & 0.9294 \\
\hline 0.35 & 0.7288 \\
\hline 0.40 & 0.6411 \\
\hline 0.45 & 0.6245 \\
\hline 0.50 & 0.6447 \\
\hline 0.60 & 0.7308 \\
\hline
\end{tabular}

TABLE 2: Amplitude of $P_{z z}$ at $z=-\delta / 2$ for the He-Xe mixture and $\delta=1$

\begin{tabular}{|c|c|}
\hline$T$ & $\left|P_{z z}(z=-\delta / 2)\right|$ \\
\hline 1.5 & 1.228 \\
\hline 2.0 & 1.323 \\
\hline 2.5 & 1.219 \\
\hline 3.0 & 1.045 \\
\hline 3.5 & 0.9143 \\
\hline 4.0 & 0.8399 \\
\hline 4.6 & 0.7998 \\
\hline 5.3 & 0.7873 \\
\hline 6.0 & 0.7978 \\
\hline 6.5 & 0.8176 \\
\hline
\end{tabular}

TABLE 3: Amplitude of $P_{z z}$ at $z=-\delta / 2$ for the He-Xe mixture and $\delta=10$

\begin{tabular}{|c|c|}
\hline$T$ & $\left|P_{z z}(z=-\delta / 2)\right|$ \\
\hline 15 & 1.194 \\
\hline 20 & 1.234 \\
\hline 25 & 1.279 \\
\hline 30 & 1.322 \\
\hline 35 & 1.362 \\
\hline 40 & 1.398 \\
\hline 45 & 1.442 \\
\hline 51 & 1.495 \\
\hline 60 & 1.515 \\
\hline
\end{tabular}

rarefaction parameters taken into account $(\delta=0.1$ and $\delta=10)$, two different forced-sound modes are simultaneously present: a fast and a slow wave. The slow wave is a damped soundlike mode primarily carried by the Xe, while the fast wave should be associated with the He component of the mixture which plays a dominant role in the appearance of resonances. For $\delta=0.1$, the fast wave appears to be a single-component gas-based sound mode since the velocity field $v_{z}^{1}$ for helium is the same as that obtained for a simple monatomic gas (Desvillettes and Lorenzani, 2012), while 

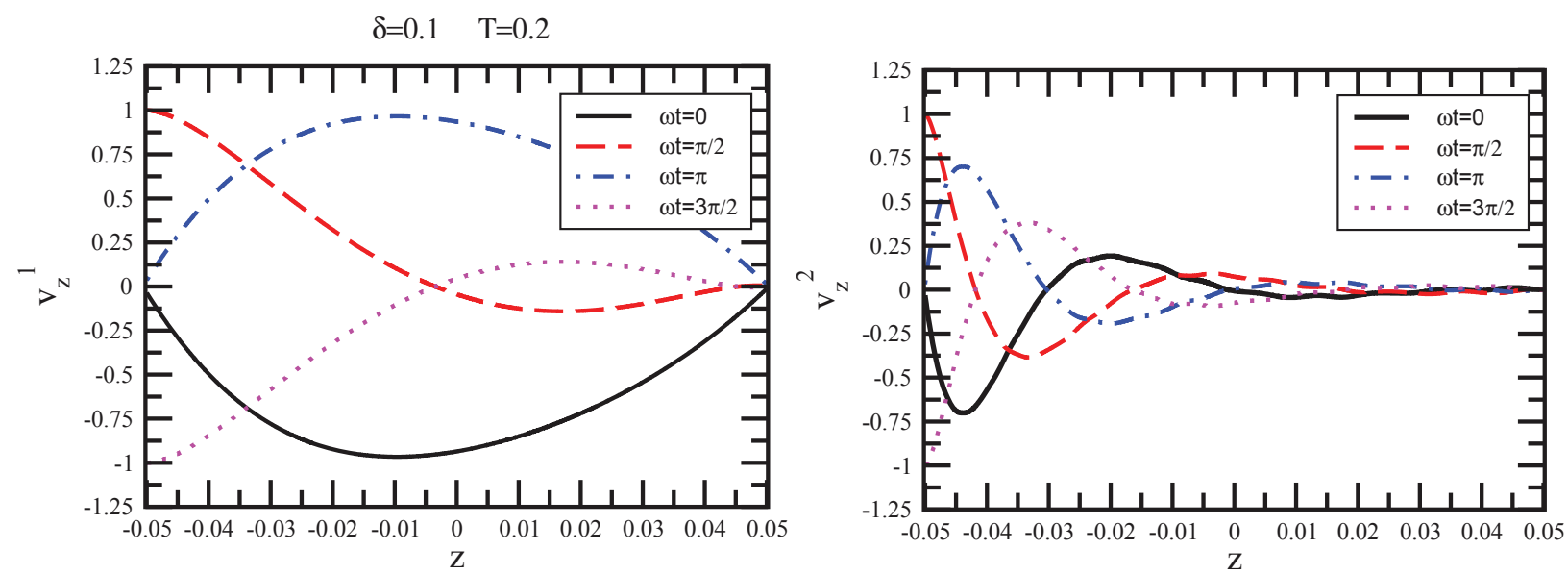

FIG. 4: Variation of the macroscopic velocities of gas components in the $z$ direction across the gap of the channel for $\delta=0.1$ and $T=0.2$.


FIG. 5: Variation of the macroscopic velocities of gas components in the $z$ direction across the gap of the channel for $\delta=10$ and $T=23$.

for $\delta=10$, the fast wave in He is not analogous to a sound wave in a pure monatomic gas. These observations are in agreement with previous predictions about the possibility of several simultaneous sound modes in a disparate-mass gas mixture at a high enough frequency (Bowler and Johnson, 1985; Huck and Johnson, 1980; Johnson, 1989), but they deserve further investigation in order to clarify precisely the nature and the origin of these different interfering modes.

\section{CONCLUDING REMARKS}

In the present paper, we have analyzed the damping forces exerted by a binary gaseous mixture in micro-electromechanical systems devices vibrating at high frequencies through the kinetic theory of rarefied gases. We have found that, due to the constructive/destructive interference occurring between the incident and reflected sound waves generated by the high-frequency oscillating boundary of the microdevice, a resonant/antiresonant response of the system can be detected in the near-free molecular flow regime and in the transitional region (similarly to the case of a single- 
component gas). Corresponding to a resonant response of the system, the amplitude of $P_{z z}$ at $z=-\delta / 2$ reaches its maximum value (resonance) or its minimum value (antiresonance). The occurrence of an antiresonance is particularly important since if the device is operated close to the corresponding frequency, the damping due to the gas is considerably reduced. However, contrary to the case of a single-component gas, our numerical results show that, in the near-continuum regime, the propagation of sound waves in the helium-xenon mixture does not show any resonance. According to our analysis, this behavior is peculiar to disparate-mass gas mixtures when anomalous dispersion of forced sound is observed (Bowler and Johnson, 1985). Therefore, we are led to interpret the different behavior of sound wave propagation in a $\mathrm{He}-\mathrm{Xe}$ mixture at $\delta=10$ as related to the existing interference between the sound mode and another excitation mode of the gas (Bowler and Johnson, 1985). To conclude, our model allows one to study the nature of the multiple forced-sound modes excited in disparate-mass gas mixtures, thanks to the peculiar characteristics shown by the sound waves in correspondence of the resonant/antiresonant frequency. In this way, it has been possible to detect that, in the free-molecular flow regime and in the transitional region (where resonances still occur), the sound mode associated with the He component of the mixture is a single component gas-based sound mode, while in the continuum limit the excited mode carried by the He component is not analogous to the sound wave propagating in a pure monatomic gas. This allows one to clarify the longstanding open question about the precise nature of sound modes excited in disparate-mass gas mixtures (Bowler and Johnson, 1985; Huck and Johnson, 1980; Johnson, 1989).

\section{ACKNOWLEDGMENT}

The authors are grateful to the GNFM for its financial support.

\section{REFERENCES}

Andries, P., Aoki, K. and Perthame, B., A consistent BGK-type model for gas mixtures, J. Stat. Phys., vol. 106, pp. 993-1018, 2002.

Bowler, J. R. and Johnson, E. A., Anomalous dispersion in disparate-mass gas mixtures, Phys. Rev. Lett., vol. 54, pp. 329-332, 1985.

Chapman, S. and Cowling, T. G., The Mathematical Theory of Non-uniform Gases, Cambridge University Press, Cambridge, 1970.

Desvillettes, L. and Lorenzani, S., Sound wave resonances in micro-electro-mechanical systems devices vibrating at high frequencies according to the kinetic theory of gases, Phys. Fluids, vol. 24, 092001, 2012.

Groppi, M. and Spiga, G., A Bhatnagar-Gross-Krook-type approach for chemically reacting gas mixtures, Phys. Fluids, 16, pp. 4273-4284, 2004.

Huck, R. J. and Johnson, E. A., Possibility of double sound propagation in disparate-mass gas mixtures, Phys. Rev. Lett., vol. 44, pp. 142-145, 1980.

Johnson, E. A., Two-temperature hydrodynamics and multiple sound modes in disparate-mass gas mixtures, J. Stat. Phys., vol. 57, pp. 647-664, 1989.

Kalempa, D. and Sharipov, F., Sound propagation through a rarefied gas confined between source and receptor at arbitrary Knudsen number and sound frequency, Phys. Fluids, vol. 21, 103601, 2009.

Kestin, J., Knierim, K., Mason, E. A., Najafi, B., Ro, S. T., and Waldman, M., Equilibrium and transport properties of the noble gases and their mixtures at low density, J. Phys. Chem. Ref. Data, vol. 13, pp. 229-303, 1984.

Kosuge, S., Aoki, K., Inoue, T., Goldstein, D. B., and Varghese, P. L., Unsteady flows in Io's atmosphere caused by condensation and sublimation during and after eclipse: Numerical study based on a model Boltzmann equation, Icarus, vol. 221, pp. 658-669, 2012.

Kosuge, S., Mizuno, H., and Aoki, K., Numerical investigation on models of the Boltzmann equation for gas mixtures, in Proc. of Rarefied Gas Dynamics: 25th Intl. Symp., (edited by Ivanov, M. S. and Rebrov, A. K.), Novosibirsk, pp. 286-291, 2007.

Lorenzani, S., Gibelli, L., Frezzotti, A., Frangi, A., and Cercignani, C., Kinetic approach to gas flows in microchannels, Nanoscale Microscale Thermophys. Eng., vol. 11, pp. 211-226, 2007.

McCormack, F. J., Construction of linearized kinetic models for gaseous mixtures and molecular gases, Phys. Fluids, vol. 16, pp. 2095-2105, 1973. 